\title{
VIEWPOINT
}

\section{Phases-of-illness paradigm: better communication, better outcomes}

\author{
Jeremy C Pamplin*1, Sarah J Murray ${ }^{2}$ and Kevin K Chung ${ }^{3}$
}

\begin{abstract}
Communication failures are a significant contributor to medical errors that harm patients. Critical care delivery is a complex system of inter-professional work that is distributed across time, space, and multiple disciplines. Because health-care education and delivery remain siloed by profession, we lack a shared framework within which we discuss and subsequently optimize patient care. Furthermore, our disparate professional perspectives and interests often interfere with our ability to effectively prioritize individual care. It is important, therefore, to develop a cognitively shared framework for understanding a patient's severity of illness and plan of care across multiple, traditionally poorly communicating disciplines. We suggest that the 'phases-of-illness paradigm' will facilitate communication about critically ill patients and create a shared mental model for interdisciplinary patient care. In so doing, this paradigm may reduce communication errors, complications, and costs while improving resource utilization and trainee education. Additional research applications are feasible.
\end{abstract}

\section{Problem}

Communication problems and errors of the interdisciplinary health-care team are vast problems affecting the safety and effectiveness of patient care; as many as $67 \%$ of sentinel events reported to the Joint Commission are directly related to communication failures [1]. In its seminal report 'To Err Is Human: Building a Safer Health System, the Institute of Medicine attributed up to 98,000 deaths as related to medical error [2], making medical error potentially the sixth leading cause of death in the US [3]. Other reports similarly indicate that medical

\footnotetext{
*Correspondence: jeremy.pamplin@us.army.mil

'Brooke Army Medical Center, 3851 Roger Brooke Dr., Fort Sam Houston,

TX 78234-6200, USA

Full list of author information is available at the end of the article
}

injuries and management mistakes account for tremendous costs in resources and dollars $[4,5]$.

Key to addressing this problem is the 'divergence' among various team members as to what the priorities are for a patient's care. 'Communication failures are often rooted in an internecine conflict between team members about these priorities.' For example:

Bedside nurse: 'We haven't done the rehab yet today.'

Attending physician: 'Why?'

Bedside nurse: 'We are waiting for ultrasound to come up.'

Attending physician: 'Let's get [the patient] up now.'

This dialog represents 'a misunderstanding of intent and a misunderstanding regarding the importance of rehabilitation therapy in [this patient's] care plan. There seems to be an intangible desire not to 'rock the boat' by elevating questions of care priorities to the level of physician and/or multidisciplinary team awareness. These subtle undercurrents of disagreements between different professionals about how to prioritize care are never discussed by the multidisciplinary team' (personal communication, Dr Leopold Cancio, US Army Institute of Surgical Research).

This clinical scenario, particularly when played out in the ICU, reflects a fundamental challenge to the multidisciplinary health-care team: getting the whole team to play from the same page of music. As a communication tool, music provides a common language to all members of an orchestra. There is no such language for a healthcare team. Communication failure originates in our 'siloed' professional system [6]. While the ICU environment may boast a multidisciplinary approach to care, in actuality the different disciplines operate independently, and their communication across multiple media often fails. What is needed is an interdisciplinary approach by which each member of the team communicates using shared expectations and a shared language about a patient's status and goals.

\section{Solution: the phases-of-illness paradigm}

We propose the phases-of-illness paradigm (POIP) as a shared mental model, or framework, within which providers may identify patients according to severity of 
illness and apply supportive therapies according to patient-oriented goals and care team objectives (Figure 1, Table 1). In each 'phase', patients have similar severities of illness, goals of care, and treatment objectives. Within this framework, checklists help identify the patient's severity of illness and priorities of care. Patients may move forward (rightward) or backward (leftward) through acute illness, stabilization, weaning, and recovery phases. Patients transitioned to palliative care would exit this model into a palliative care 'phase' that is distinct because it is blind to a patient's severity of illness. Most clinicians implicitly utilize a similar model to help them recognize a patient's condition and to target a care plan. POIP explicitly states these goals and objectives so that all team members may recognize and share them.

The POIP has the advantages of several key quality improvement tools, including clinical pathways, bundles of care, and checklists. Each of these tools has been demonstrated to reduce complications, reduce cost, and improve outcomes [7-9]. Common to each of these tools is the notion of shared responsibility and objective assessments of care quality. Once the entire care team recognizes a patient's severity of illness, it can share the responsibility of ensuring that the care plan is optimized to achieve explicitly stated goals by reviewing checklists (a type of cognitive aid). Any member of the team can identify areas for improvement, and the team may quantify successful care by its achievement of goals. Sharing the responsibility of goal attainment for supportive care across the care team reduces individual cognitive load, thus freeing cognition for other, potentially more important functions, such as making an accurate diagnosis, optimizing a treatment strategy, providing valuable family support, or attending another patient.

\section{Application}

A patient's progress through the ICU can be thought of as a continuum in which movement may occur both forward and backward. A 'phase of illness' is a distinct period along the continuum in which patients with similar characteristics may be grouped or stratified. In each phase, patients' severity of illness and the healthcare teams' goals of care are similar for all the patients in that phase (Table 1). For example, we describe the most acute or sickest stratum as the 'acute' phase. During this phase, we prioritize life-saving therapies and effective organ support. Thus, a patient's phase determines our goal priorities. Within each phase, it is simple to create phase-specific checklists for your institution that help ensure adherence to local protocols, best practices, clinical guidelines, or specific care bundles. Similarly, order sets may be written to facilitate patient-driven standardization of supportive care elements, such as types of monitoring, frequency and type of laboratory assessment, sedation strategies, modes of mechanical ventilation, and physical therapy interventions (Figure 1).

Although this framework offers a shared mental model of patient care to the interdisciplinary team, not all team members interact with it in the same way. The experienced provider, for example, conceptualizes patient movement through phases intuitively. This intuition is best expressed by a provider changing goals of care for a patient. The advantage of the POIP for the inexperienced health-care provider is the ability to categorize patients according to criteria and to have a roadmap for recovery. Often, the inexperienced provider gets 'stuck' providing the same level of care to a patient and is unable to recognize when to transition goals. For this provider, it is useful to ask, 'What needs to be done to move to the next phase?' If the patient meets certain criteria, such as 'no longer in shock', the inexperienced provider may be more comfortable advancing the patient from an acute illness phase to a stabilization phase and then can utilize the goals within the stabilization phase to establish a plan of care.

For both experienced and inexperienced health-care providers, adding an additional 'pause' phase (which we call a 'pause cloud') is also useful. A pause occurs when a clinician recognizes a potential change in patient condition that requires additional monitoring or investigation. It occurs when a patient who was previously advancing in his or her care - tolerating weaning, diuresing, demonstrating improved mental status, and so on - 'pauses' in this progress for unclear reasons. In this event, the team recognizes that the change may or may not be significant but that it requires additional evaluation. A pause may be brief (for example, awaiting a lab result) or may be more prolonged ('let's not walk the patient today; he looks like he may be getting sicker'). The key, however, is that the patient has not gotten worse and remains within his or her current phase. If, during this heightened period of observation, the patient's condition deteriorates, the goals of care change, and the patient regresses to a different phase of illness that better reflects his severity of illness. Conversely, if his condition does not worsen during the pause, he continues to progress forward through the continuum of care.

\section{Strengths and weaknesses}

The complexities of critical care are beyond the capacity of one provider or a single professional group to deliver optimal patient care [10]. The emergence of the multidisciplinary care team as an optimal model for care delivery is well supported by the literature [11]. Unfortunately, the shared responsibilities of patient care require frequent negotiations between team members in order to deliver optimal care. In our current culture, most disciplines approach disagreement by using positional bargaining strategies to direct patient care: 


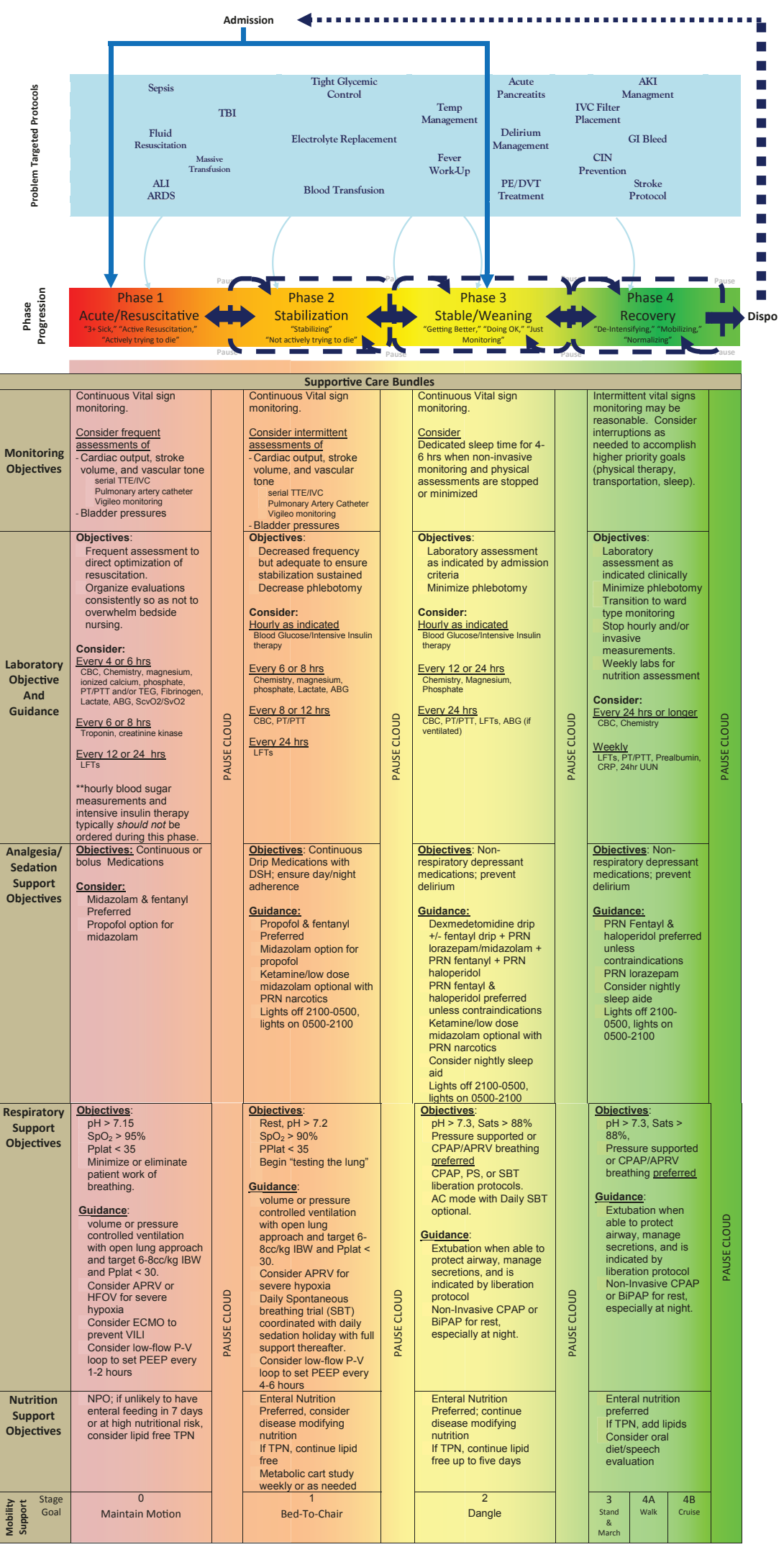

Figure 1. See overleaf for legend. 


\begin{abstract}
Figure 1. The phases-of-illness paradigm. Patients enter the ICU environment for one of two reasons: resuscitation (organ support, including respiratory failure, shock states, acute liver failure, and so on) or ICU monitoring. Patients who need resuscitation are in shock and need aggressively titrated and carefully monitored care. Patients who need monitoring are typically 'stable', but need a higher level of observation than is available elsewhere in the hospital: hourly checks or interventions, invasive monitors, and so on. Movement through the continuum of phases is fluid, timeless, and directionless. A patient getting better will move to the right and a patient getting worse to the left. Since a severity of illness may describe any type of patient, and supportive care goals (Table 1) apply to all patients with a certain severity of illness, additional 'disease-specific' protocols may also apply to a patient. Phase specific protocols or checks in this table are examples only: these objectives and do-confirm type checks must be adapted to fit a local environment and culture. The 'pause cloud' is an 'in-between' phase during which it is unclear what 'direction' a patient is moving (that is, could be getting better or worse). Typically, monitoring may increase, decrease, or stay the same as the patient's current phase. Sometimes this phase may be a brief 'check' (check another set of labs, check an imaging study, check cultures, and so on). Sometimes it may be more prolonged (for example, during traumatic brain injury (TBI) when intracranial pressure (ICP) management is ongoing but unchanging). ABG, arterial blood gas; AC mode, pressure or volume assist control mode of ventilation; AKI, acute kidney injury; ALI, acute lung injury; APRV, airway pressure release ventilation; ARDS, acute respiratory distress syndrome; BiPAP, bi-level pressure consisting of inspiratory and expiratory positive airway pressure; CBC, complete blood count; CIN, contrast-induced nephropathy; CPAP, continuous positive airway pressure; CRP, C-reactive protein; DSH, daily sedation holiday; DVT, deep vein thrombosus; ECMO, extracorporeal membrane oxygenation; Gl, gastrointestinal; HFOV, high frequency oscillatory ventilation; IBW, ideal body weight; ICP, intracranial pressure; IVC, inferior vena cava; LFT, liver functions test; NPO, noting per os (nothing to eat by mouth); PE, pulmonary embolism; PEEP, positive end expiratory pressure; Pplat, plateau pressure; PRN, as needed; PS, pressure support; PT/PTT, prothrombin time/partial thromboplastin time; P-V loop, pressure volume loop; SBT, spontaneous breathing trial; ScvO2/SvO2, central vein oxygen saturation/mixed venous oxygen saturation; SpO2, peripheral oxygen saturation; TBI, traumatic brain injury; TEG, thromboelastogram; TPN, total parenteral nutrition; TTE, transthoracic echocardiogram; UUN, urine urea nitrogen; VILI, ventilator induced lung injury.
\end{abstract}

Provider 1: 'I want the patient to have 2 units of blood.' Provider 2: 'Why?'

Provider 1: 'Because I think it will help him feel better'. Provider 2: 'There's no evidence for that.'

Provider 1: 'Do it anyway.'

According to the theory of principled negotiation, objective measures are needed to determine how well a course of action satisfies the interests of the parties involved in the negotiation [12]. The POIP provides these objective measures by establishing patient goals. To aid these negotiations, goals need to be identified as patientcentric needs that may be addressed through multiple possible solutions; objectives are the different solutions that the health-care team may take, and tasks are the responsibilities of individuals in the team required to achieve an objective (Table 2). Using the POIP to facilitate principled negotiation, the negotiation in the previous scenario for a patient in the recovery phase might become the following:

Provider 1: 'I want the patient to have 2 units of blood.'

Provider 2: 'What goal will that help us achieve?'

Provider 1: 'I think it will help him achieve his physical therapy goals today because he failed them yesterday.

While POIP provides a framework for the health-care team to better communicate, it may also facilitate communication with patients and family members. Patients and their families have difficulty understanding the direction of their progress through the ICU, and the health-care team often has difficulty explaining it to them. A framework that groups severity of illness and care goals helps families to understand where in the continuum of care they are and where they need to go to get better. It helps them to focus on goals of care, less on how to achieve them, and thus facilitates the shared decision-making model [13]. Finally, the POIP provides transparency to our discussions with a patient or family by giving them a visual depiction of how they move through critical illness.

The future applications of a phase-based paradigm in the ICU are robust. In particular, administrative and research benefits may be significant. Currently, extensive resources are spent understanding and managing patient throughput in a hospital and matching resources, particularly human resources (most notably nursing), to patient needs. After adopting a phase-based paradigm, supervising bed managers could check the status of an ICU and rapidly determine potential changes in capacity. If a ten-bed ICU has five patients in the acute phase of illness, it is unlikely that these patients will leave the ICU in the next 24 to 48 hours unless they die. If there are three 'recovery' phase patients in the remaining beds, the managers' time and efforts may be placed on determining what resources are needed to facilitate those patients' transfer out of the ICU. The remaining patients would require little attention from the bed manager. Furthermore, the resource manager may better match staffing to patient needs. It is likely that a patient in the acute illness phase will need more one-to-one nursing than a patient in the recovery phase.

Future research applications of the POIP will need to focus on how it affects patient care. Because the POIP stratifies patients according to clinical condition, studies of ICU populations could prospectively stratify patients because therapies of interest may be more or less effective at a particular time during a patient's ICU course (for example, red blood cell transfusions). Research questions specific to this paradigm must also be answered: does the POIP improve communication and thus reduce errors 
Table 1. Example of the phases-of-illness paradigm phase criteria and supportive care goals

\begin{tabular}{|c|c|}
\hline & $\begin{array}{l}\text { Phase } 1 \text { - Acute } \\
\text { (6 to } 24 \text { hours; few patients) }\end{array}$ \\
\hline \multirow[t]{12}{*}{ Criteria } & Presence of shock \\
\hline & SBP $<90$ after fluid bolus \\
\hline & An elevated lactate (>2 to 4 ) \\
\hline & $\begin{array}{l}\text { Decreased } \mathrm{SvO} 2 \text { or } \mathrm{ScvO} 2 \\
(<70 \% \text { or }<65 \%)\end{array}$ \\
\hline & $\begin{array}{l}\text { Active resuscitation: medications, } \\
\text { drips, and therapies are rapidly } \\
\text { added and/or changed }\end{array}$ \\
\hline & $\begin{array}{l}\text { Rapid fluid/blood product } \\
\text { infusions }\end{array}$ \\
\hline & $\begin{array}{l}\text { Respiratory failure and need for } \\
\text { advanced mechanical ventilation }\end{array}$ \\
\hline & $\begin{array}{l}\text { Increasing extracorporeal life } \\
\text { support for organ failure }\end{array}$ \\
\hline & Aggressive ICP management \\
\hline & $\begin{array}{l}\text { Frequent osmolar therapy, CSF } \\
\text { drainage, pending need for } \\
\text { craniectomy }\end{array}$ \\
\hline & $\begin{array}{l}\text { Initial } 8 \text { to } 12 \text { hours of burn } \\
\text { resuscitation }\end{array}$ \\
\hline & The need for organ support \\
\hline
\end{tabular}

Goals

Phase 2 - Stabilization ( 2 to 4 hours, often days in TBI)

Phase 3 - Stable/weaning

SvO2 or ScvO2 has normalized

Lactate has normalized or continues to decrease

Stable vasopressor requirement for 2 to 4 hours

No new therapies for 2 to 4 hours No fluid bolus for 2 to 4 hours Stable ventilator settings, but unable to wean (not on extracorporeal lung support) Not on high volume CRRT

Continued ICP elevations requiring intervention more than every 12 hours

8 to 36 hours of burn resuscitation

The need for organ support

Stable

\section{Stable}

Admitted to or in the ICU primarily for monitoring purposes

One-to-one nursing, or invasive monitoring not supported elsewhere in the hospital

\section{Weaning}

The patient is getting better Vasoactive drips are being decreased or weaned off Ventilator settings are being weaned or modes changed to CPAP or pressure support

Possible extubation

Active or passive dieresis Renal support CRRT Reducing the need for organ support
Phase 4 - Recovery (indefinite; most patients)

No vasoactive drips

Diuresing or euvolemia

Liberated or attempting to liberate from mechanical ventilation or unable to liberate due to long-term condition

Invasive devices are being removed.

The patient is being mobilized (PT/OT)

Transition to intermittent hemodialysis

No need for organ support

The need for organ support

General supportive

Stable

To continue resuscitation as needed, likely with less frequent interventions

To maintain stabilization achieved example, acute lung injury) resuscitation

To assess and maintain vital organ perfusion by:

Volume resuscitating

Maintaining adequate perfusion pressure with continuous vasopressor medications

Achieve a normal ScvO2 or $\mathrm{SvO} 2$ and/or a down trending lactate

To achieve adequate gas exchange to perfuse organs utilizing advanced mechanical ventilation and or extracorporea support therapies

To prevent uncal herniation and brain death

Analgesia/ Controlled sedation for: safety,

sedation rest, and decreased metabolic

support demand. Avoid sedative-induced hypotension

Assess neurologic function with awakening trial/re-establish awareness. Maintain and prioritize pain control

Ventilation Safety, rest, and control; avoid VILI

Respiratory work to avoid atrophy; avoid VILI

Facilitate awareness, change position to minimize atelectasis
To closely monitor the patient for potential worsening of a specific condition such as: Neurologic decline Respiratory distress Extremity compromise (vascular/neurologic) Hemodynamic compromise

Weaning

To decrease resuscitative support

Wean-off vasopressor therapy

Decrease/remove IVF

Wean ventilator support

Reduce organ support

Both

Minimize cognitive harm and physical wasting Interaction

A pain-free, awake, and interactive patient that can participate in care. Avoid respiratory depressants

Comfortable spontaneous breathing. Possible extubation

Re-establish postural tone. Maintain strength
To remove invasive devices Cannula(e), ETT, central line, arterial line, Foley, and so on To ready the patient for transfer or discharge

Discontinue continuous drips

Decrease monitoring

Stopping ICU-related

medications (such as stress ulcer prophylaxis, intensive insulin therapy, and so on)

Reestablish patient self-control and function

Pain-free and participative in care Transition to as needed, preferably oral/enteral medication

Comfortable spontaneous breathing, preferably without an endotracheal tube (that is, NIPPV or tracheostomy)

Rehabilitation, independence

Either criteria or goals of care may define a patient's phase of illness. Experienced providers typically conceptualize a patient's care goals first, whereas inexperienced providers typically need to identify a patient's severity of illness first and then define the goals of care. The tool is especially useful to inexperienced providers and interdisciplinary teams because it provides a conceptual 'roadmap' of patient progress, similar to a clinical pathway. CPAP, continuous positive airway pressure; CRRT, continuous renal replacement therapy; CSF, cerebrospinal fluid; DVT, deep venous thrombosis; ETT, endotracheal tube; ICP, intracranial pressure; IVF, intravenous fluid; NIPPV, non-invasive positive pressure ventilation; PT/OT, physical therapy/occupational therapy; SBP, systolic blood pressure; ScvO , central venous oxygen saturation; $\mathrm{SvO}_{2}$, mixed venous oxygen saturation; $\mathrm{TBI}$, traumatic brain injury; $\mathrm{VILI}$, ventilator-induced lung injury. 
Table 2. Nomenclature for goals, objectives, and tasks as related to patient care

\begin{tabular}{llll}
\hline & Goal & Objective & Task \\
\hline Focus & Patient & Healthcare team & Responsible individual \\
Strategies & Multiple possibilities & Defined options & 1. Single option \\
Example & Stop organ failure & Resuscitate to $>10$ & 1. Notify physician if CVP $<10$ (nurse) \\
& & 2. Order bolus for CVP $<10$ (ILP) \\
& VAP $>60$ & 3. Titrate norepinephrine to MAP $>60$ (nurse) \\
& Breathing trial daily & 1. Order ventilator liberation protocol (ILP) \\
& & 2. Hold continuous sedation at 0600 (nurse) \\
& & 3. Notify RT when the patient is'awake' (nurse) \\
\hline
\end{tabular}

CVP, central venous pressure; ILP, independent licensed practitioner; MAP, mean arterial pressure; RT, respiratory therapist.

Table 3. Suggested evaluation questions for designing an effective ICU daily checklist

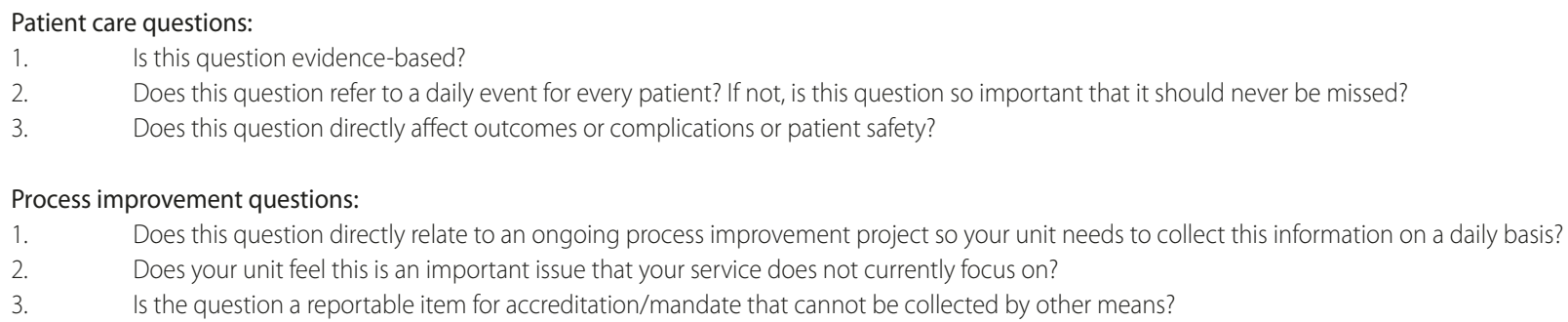

and improve patient safety? Will use of this paradigm improve patient outcomes? Can use of this paradigm decrease costs? Can this paradigm be used as a predictive model for patient outcomes?

There are several potential concerns when adopting the POIP. First, there are always patients who do not fit well into any model. When a patient's phase is unclear or there is disagreement about a patient's phase, we recommend focusing on the goals of care, not the phase criteria. In general, focusing on the goals will help determine whether a patient is 'sicker' or 'less sick' and can better define the patient's current phase. If phase determination still proves challenging, it is simple to make a choice and pursue phase goals that make the most sense for the patient. In this case, a group's conservative or aggressive biases often dictate the phase choice. The key is to make a decision so that all team members can understand a patient's status and then move forward with care.

Second, the model's transparency with respect to patient severity of illness may lead some members of the team, particularly family members, to lose hope. For example, a spouse may feel fear or hopelessness when his wife, who was doing very well in a recovery phase, moves to a resuscitation phase. It is important to recognize that although this paradigm defines a desired trajectory through the ICU, it should not be used to predict patient outcome. A phase only describes a patient's current condition so that the entire team, including the family and the patient, may better understand expectations and therefore have a better framework for communication and planning. While establishing and maintaining a trajectory through this model is the goal, falling back to prior phases does not prohibit reaching the final goal of recovery.

Third, it is vital for an institution to closely examine the model and adapt it, particularly the phase-based checklists and order sets, to the local environment. Phase criteria and goals that may fit well for one ICU may not fit well for another (Table 3).

\section{Conclusion}

Overall, we believe that the POIP will enhance the ability of the entire multidisciplinary health-care team to more effectively recognize a patient's changing condition, establish a patient-driven care plan, and communicate with each other about patient care. Establishing daily goals for patients according to their condition and delivering specific treatments according to this condition in a patient-centric model will decrease time and money spent in the ICU. Additional improvements in resource utilization and research applications are also possible. Ultimately, aligning a patient's condition with specific goals and therefore treatments in a phase-based paradigm will help all health-care providers choose the most appropriate therapies to facilitate a patient's recovery.

\section{Abbreviations}

POIP, phases-of-illness paradigm.

\section{Competing interests}

The authors declare that they have no competing interests. 


\section{Authors' contributions}

$J C P$ is the originator of the concept, wrote, and edited and approved the final manuscript. SJM contributed intellectual content, wrote portions of the manuscript, and edited and approved the final manuscript. KKC contributed intellectual content and edited and approved the final manuscript.

\section{Acknowledgements}

Ms Otilia Sánchez assisted with editing multiple versions of the manuscript. The authors are very appreciative of her time and expertise.

The opinions or assertions contained herein are the private views of the author(s) and are not to be construed as official or as reflecting the views of the Department of the United States Army or the United States Department of Defense.

\section{Author details}

'Brooke Army Medical Center, 3851 Roger Brooke Dr., Fort Sam Houston, TX 78234-6200, USA. ${ }^{2} 481$ D Kawailoa Road, Kailua, HI 96734, USA. ${ }^{3}$ US Army Institute of Surgical Research, 3698 Chambers Pass, Fort Sam Houston, TX 78234-6200, USA

\section{Published: 28 November 2011}

\section{References}

1. The Joint Commission: Sentinel event root cause and trend data. In Improving America's Hospitals: The Joint Commission's Annual Report on Quality and Safety. Nov 2007:46-47. [http://www.jointcommissionreport.org]

2. Kohn LT, Corrigan JM, Donaldson MS: To Err is Human: Building a Safer Health System. Washington DC: The National Academies Press; 2000

3. Xu J, Kochanek KD, Murphy SL, Tejada-Vera Betzaida: Deaths: Final data for 2010. Natl Vital Stat Rep 2010, 58:1-134.

4. Stahl K, Palileo A, Schulman CI, Wilson K, Augenstein J, Kiffin C, McKenney M: Enhancing patient safety in the trauma/surgical intensive care unit. J Trauma 2009, 67:430-433: discussion 433-435.

5. Chunliu Z, Miller MR: Excess length of stay, charges, and mortality attributable to medical injuries during hospitalization. JAMA 2003, 290:1868-1874

6. Hall P: Interprofessional teamwork: professional cultures as barriers. $J$ Interprof Care 2005, 19 Suppl 1:188-196.
7. Girard TD, Kress JP, Fuchs BD, Thomason JW, Schweickert WD, Pun BT, Taichman DB, Dunn JG, Pohlman AS, Kinniry PA, Jackson JC, Canonico AE, Light RW, Shintani AK, Thompson JL, Gordon SM, Hall JB, Dittus RS, Bernard GR, Ely EW: Efficacy and safety of a paired sedation and ventilator weaning protocol for mechanically ventilated patients in intensive care (awakening and breathing controlled trial): A randomised controlled trial. Lancet 2008, 371:126-134.

8. Zevola DR, Raffa M, Brown K: Using clinical pathways in patients undergoing cardiac valve surgery. Crit Care Nurse 2002, 22:31-9, 44-50.

9. Levy MM, Dellinger RP, Townsend SR, Linde-Zwirble WT, Marshall JC, Bion J, Schorr C, Artigas A, Ramsay G, Beale R, Parker MM, Gerlach H, Reinhart K, Silva E, Harvey M, Regan S, Angus DC; Surviving Sepsis Campaign: The Surviving Sepsis Campaign: Results of an international guideline-based performance improvement program targeting severe sepsis. Crit Care Med 2010, 39:367-374.

10. Plsek PE, Greenhalgh T: Complexity science: The challenge of complexity in health care. BMJ 2001, 323:625-628.

11. Brilli RJ, Spevetz A, Branson RD, Campbell GM, Cohen H, Dasta JF, Harvey MA Kelley MA, Kelly KM, Rudis MI, St Andre AC, Stone JR, Teres D, Weled BJ; American College of Critical Care Medicine Task Force on Models of Critical Care Delivery. The American College of Critical Care Medicine Guidelines for the Defintion of an Intensivist and the Practice of Critical Care Medicine: Critical care delivery in the intensive care unit: defining clinical roles and the best practice model. Crit Care Med 2001, 29:2007-2019.

12. Fisher R, Ury WL, Patton B: Getting to Yes: Negotiating Agreement Without Giving In. 3rd edition. New York: Penguin; 2011.

13. Davidson JE, Powers K, Hedayat KM, Tieszen M, Kon AA, Shepard E, Spuhler V Todres ID, Levy M, Barr J, Ghandi R, Hirsch G, Armstrong D; American College of Critical Care Medicine Task Force 2004-2005, Society of Critical Care Medicine: Clinical practice guidelines for support of the family in the patient-centered intensive care unit: American College of Critical Care Medicine Task Force 2004-2005. Crit Care Med 2007, 35:605-622.

doi:10.1186/cc10335

Cite this article as: Pamplin JC, et al.: Phases-of-illness paradigm: better communication, better outcomes. Critical Care 2011, 15:309. 